\title{
Higher-Order Commutators of Parametric Marcinkiewicz Integrals on Herz Spaces with Variable Exponent
}

\author{
Hongbin Wang $\mathbb{D}^{1,2}$ and Dunyan Yan $\mathbb{D}^{2}$ \\ ${ }^{1}$ School of Mathematics and Statistics, Shandong University of Technology, Zibo 255049, China \\ ${ }^{2}$ School of Mathematical Sciences, University of Chinese Academy of Sciences, Beijing 100049, China \\ Correspondence should be addressed to Hongbin Wang; wanghb@sdut.edu.cn
}

Received 27 September 2018; Accepted 16 October 2018; Published 1 November 2018

Academic Editor: Alberto Fiorenza

Copyright (c) 2018 Hongbin Wang and Dunyan Yan. This is an open access article distributed under the Creative Commons Attribution License, which permits unrestricted use, distribution, and reproduction in any medium, provided the original work is properly cited.

Let $\Omega \in L^{s}\left(S^{n-1}\right)$ for $s \geqslant 1$ be a homogeneous function of degree zero and $b$ be BMO functions. In this paper, we obtain some boundedness of the parametric Marcinkiewicz integral operator $\mu_{\Omega}^{\rho}$ and its higher-order commutator $\left[b^{m}\right.$, $\left.\mu_{\Omega}^{\rho}\right]$ on Herz spaces with variable exponent.

\section{Introduction}

Function spaces with variable exponent are being concerned with strong interest not only in harmonic analysis but also in applied mathematics. In the past 27 years, the theory of function spaces with variable exponent has made great progress since some elementary properties were given by Kováčik and Rákosník [1] in 1991. In [2-6], the authors proved the boundedness of some integral operators on variable $L^{p}$ spaces, respectively. Lebesgue and Sobolev spaces with integrability exponent have been widely studied; see $[3,5]$ and the references therein. Many applications of these spaces were given, for example, in the modeling of electrorheological fluids, in the study of image processing, and in differential equations with nonstandard growth.

On the other hand, a class of function spaces called Herztype spaces on $\mathbb{R}^{n}$ has attracted considerable attention in recent years because the interesting norm includes explicitly both local and global information of the function. In 2011, Izuki [7] studied the Herz spaces with variable exponent and proved the boundedness of some sublinear operators on the spaces. In addition, Wang and Liu [8] introduced a certain Herz-type Hardy spaces with variable exponent in 2012.

Suppose that $S^{n-1}$ denotes the unit sphere in $\mathbb{R}^{n}(n \geqslant$ 2) equipped with normalized Lebesgue measure. Let $\Omega \in$
$\operatorname{Lip}_{\beta}\left(\mathbb{R}^{n}\right)$ for $0<\beta \leqslant 1$ be a homogeneous function of degree zero and

$$
\int_{S^{n-1}} \Omega\left(x^{\prime}\right) d \sigma\left(x^{\prime}\right)=0
$$

where $x^{\prime}=x /|x|$ for any $x \neq 0$. In 1958, Stein [9] introduced the Marcinkiewicz integral related to the Littlewood-Paley $g$ function on $\mathbb{R}^{n}$ as follows:

$$
\mu_{\Omega}(f)(x)=\left(\int_{0}^{\infty}\left|F_{\Omega, t}(f)(x)\right|^{2} \frac{d t}{t^{3}}\right)^{1 / 2},
$$

where

$$
F_{\Omega, t}(f)(x)=\int_{|x-y| \leqslant t} \frac{\Omega(x-y)}{|x-y|^{n-1}} f(y) d y .
$$

It was shown that $\mu_{\Omega}$ is of type $(p, p)$ for $1<p \leqslant 2$ and of weak type $(1,1)$.

The parametric Marcinkiewicz integral is defined by

$$
\mu_{\Omega}^{\rho}(f)(x)=\left(\int_{0}^{\infty}\left|F_{\Omega, t}^{\rho}(f)(x)\right|^{2} \frac{d t}{t^{2 \rho+1}}\right)^{1 / 2}
$$


where

$$
F_{\Omega, t}^{\rho}(f)(x)=\int_{|x-y| \leqslant t} \frac{\Omega(x-y)}{|x-y|^{n-\rho}} f(y) d y,
$$

$$
\rho>0, t>0 .
$$

Let $m \in \mathbb{N}$ and $b \in L_{\text {loc }}^{1}\left(\mathbb{R}^{n}\right)$, the higher-order commutator generated by the parametric Marcinkiewicz integral $\mu_{\Omega}^{\rho}$ and $b$ is defined by

$$
\begin{aligned}
& {\left[b^{m}, \mu_{\Omega}^{\rho}\right](f)(x)} \\
& =\left(\int_{0}^{\infty}\left|\int_{|x-y| \leqslant t} \frac{\Omega(x-y)}{|x-y|^{n-\rho}}[b(x)-b(y)]^{m} f(y) d y\right|^{2} \frac{d t}{t^{2 \rho+1}}\right)^{1 / 2} .
\end{aligned}
$$

Motivated by $[10,11]$, we will study the boundedness for the parametric Marcinkiewicz integral operator $\mu_{\Omega}^{\rho}$ and its commutator $\left[b^{m}, \mu_{\Omega}^{\rho}\right]$ on the Herz spaces with variable exponent, where $\Omega \in L^{s}\left(S^{n-1}\right)$ for $s \geqslant 1$.

Throughout this paper, we denote the Lebesgue measure and the characteristic function of a measurable set $A \subset \mathbb{R}^{n}$ by $|A|$ and $\chi_{A}$, respectively. The notation $f \approx g$ means that there exist constants $C_{1}, C_{2}>0$ such that $C_{1} g \leqslant f \leqslant C_{2} g$. Let $B_{k}=\left\{x \in \mathbb{R}^{n}:|x| \leqslant 2^{k}\right\}$ and $A_{k}=B_{k} \backslash B_{k-1}$ for $k \in \mathbb{Z}$. Denote $\mathbb{Z}_{+}$and $\mathbb{N}$ as the sets of all positive and nonnegative integers, $\chi_{k}=\chi_{A_{k}}$ for $k \in \mathbb{Z}, \tilde{\chi}_{k}=\chi_{k}$ if $k \in \mathbb{Z}_{+}$and $\tilde{\chi}_{0}=\chi_{B_{0}}$. In addition, $\delta_{1}$ and $\delta_{2}$ are the same as in Lemma 4.

\section{Preliminaries}

Firstly we give some notation and basic definitions on variable Lebesgue spaces. Given an open set $E \subset \mathbb{R}^{n}$, and a measurable function $p(\cdot): E \longrightarrow[1, \infty) . p^{\prime}(\cdot)$ is the conjugate exponent defined by $p^{\prime}(\cdot)=p(\cdot) /(p(\cdot)-1)$.

Define $\mathscr{P}^{0}(E)$ to be set of $p(\cdot): E \longrightarrow(0, \infty)$ such that

$$
\begin{aligned}
& p^{-}=\operatorname{essinf}\{p(x): x \in E\}>0, \\
& p^{+}=\operatorname{ess} \sup \{p(x): x \in E\}<\infty .
\end{aligned}
$$

The set $\mathscr{P}(E)$ consists of all $p(\cdot): E \longrightarrow[1, \infty)$ satisfying

$$
\begin{aligned}
& p^{-}=\operatorname{essinf}\{p(x): x \in E\}>1, \\
& p^{+}=\operatorname{ess} \sup \{p(x): x \in E\}<\infty .
\end{aligned}
$$

By $L^{p(\cdot)}(E)$ we denote the space of all measurable functions $f$ on $E$ such that for some $\lambda>0$,

$$
\int_{E}\left(\frac{|f(x)|}{\lambda}\right)^{p(x)} d x<\infty .
$$

This is a Banach function space with respect to the Luxemburg-Nakano norm

$$
\|f\|_{L^{p()}(E)}=\inf \left\{\lambda>0: \int_{E}\left(\frac{|f(x)|}{\lambda}\right)^{p(x)} d x \leqslant 1\right\} .
$$

The space $L_{\text {loc }}^{p(\cdot)}(\Omega)$ is defined by

$$
\begin{aligned}
& L_{\text {loc }}^{p(\cdot)}(\Omega):=\{f: f \\
& \left.\quad \in L^{p(\cdot)}(E) \text { for all compact subsets } E \subset \Omega\right\} .
\end{aligned}
$$

Let $f \in L_{\text {loc }}^{1}\left(\mathbb{R}^{n}\right)$; the Hardy-Littlewood maximal operator is defined by

$$
M f(x)=\sup _{r>0} \frac{1}{\left|B_{r}(x)\right|} \int_{B_{r}(x)}|f(y)| d y,
$$

where $B_{r}(x)=\left\{y \in \mathbb{R}^{n}:|x-y|<r\right\}$. The set $\mathscr{B}\left(\mathbb{R}^{n}\right)$ consists of $p(\cdot) \in \mathscr{P}\left(\mathbb{R}^{n}\right)$ satisfying the condition that $M$ is bounded on $L^{p(\cdot)}\left(\mathbb{R}^{n}\right)$

In variable $L^{p}$ spaces there are some important lemmas as follows.

Lemma 1 (see [2]). If $p(\cdot) \in \mathscr{P}\left(\mathbb{R}^{n}\right)$ and satisfies

$$
|p(x)-p(y)| \leqslant \frac{C}{-\log (|x-y|)}, \quad|x-y| \leqslant \frac{1}{2}
$$

and

$$
|p(x)-p(y)| \leqslant \frac{C}{\log (|x|+e)}, \quad|y| \geqslant|x|,
$$

then $p(\cdot) \in \mathscr{B}\left(\mathbb{R}^{n}\right)$; that is, the Hardy-Littlewood maximal operator $M$ is bounded on $L^{p(\cdot)}\left(\mathbb{R}^{n}\right)$.

Lemma 2 (see [1] generalized Hölder inequality). Let $p(\cdot) \in$ $\mathscr{P}\left(\mathbb{R}^{n}\right)$. If $f \in L^{p(\cdot)}\left(\mathbb{R}^{n}\right)$ and $g \in L^{p^{\prime}(\cdot)}\left(\mathbb{R}^{n}\right)$, then $f g$ is integrable on $\mathbb{R}^{n}$ and

$$
\int_{\mathbb{R}^{n}}|f(x) g(x)| d x \leqslant r_{p}\|f\|_{L^{p(\cdot)}\left(\mathbb{R}^{n}\right)}\|g\|_{L^{p^{\prime}(\cdot)}\left(\mathbb{R}^{n}\right)},
$$

where

$$
r_{p}=1+\frac{1}{p^{-}}-\frac{1}{p^{+}}
$$

Lemma 3 (see [7]). Suppose $q(\cdot) \in \mathscr{B}\left(\mathbb{R}^{n}\right)$. Then there exists a constant $C>0$ such that for all balls $B$ in $\mathbb{R}^{n}$,

$$
\frac{1}{|B|}\left\|\chi_{B}\right\|_{L^{q(\cdot)}\left(\mathbb{R}^{n}\right)}\left\|\chi_{B}\right\|_{L^{q^{\prime}(\cdot)\left(\mathbb{R}^{n}\right)}} \leqslant C .
$$

Lemma 4 (see [7]). Let $q(\cdot) \in \mathscr{B}\left(\mathbb{R}^{n}\right)$. Then there exists a positive constant $C$ such that for all balls $B$ in $\mathbb{R}^{n}$ and all measurable subsets $S \subset B$,

$$
\begin{gathered}
\frac{\left\|\chi_{S}\right\|_{L^{q(\cdot)\left(\mathbb{R}^{n}\right)}}}{\left\|\chi_{B}\right\|_{L^{q(\cdot)}\left(\mathbb{R}^{n}\right)}} \leqslant C\left(\frac{|S|}{|B|}\right)^{\delta_{1}} \\
\text { and } \frac{\left\|\chi_{S}\right\|_{L^{q^{(\cdot)}\left(\mathbb{R}^{n}\right)}}}{\left\|\chi_{B}\right\|_{L^{q^{\prime}(\cdot)\left(\mathbb{R}^{n}\right)}}} \leqslant C\left(\frac{|S|}{|B|}\right)^{\delta_{2}},
\end{gathered}
$$

where $\delta_{1}, \delta_{2}$ are constants with $0<\delta_{1}, \delta_{2}<1$. 
Next we recall the definition of the Herz spaces with variable exponent.

Definition 5 (see [7]). Let $\alpha \in \mathbb{R}, 0<p \leqslant \infty$, and $q(\cdot) \in$ $\mathscr{P}\left(\mathbb{R}^{n}\right)$. The homogeneous Herz space $\dot{K}_{q(\cdot)}^{\alpha, p}\left(\mathbb{R}^{n}\right)$ consists of all $f \in L_{\text {loc }}^{q(\cdot)}\left(\mathbb{R}^{n} \backslash\{0\}\right)$ such that

$$
\|f\|_{\dot{K}_{q(\cdot)}^{\alpha, p}\left(\mathbb{R}^{n}\right)}=\left\{\sum_{k=-\infty}^{\infty} 2^{k \alpha p}\left\|f \chi_{k}\right\|_{L^{q(\cdot)}\left(\mathbb{R}^{n}\right)}^{p}\right\}^{1 / p}<\infty .
$$

The nonhomogeneous Herz space $K_{q(\cdot)}^{\alpha, p}\left(\mathbb{R}^{n}\right)$ is defined as the set of all $f \in L_{\text {loc }}^{q(\cdot)}\left(\mathbb{R}^{n}\right)$ such that

$$
\|f\|_{K_{q(\cdot)}^{\alpha, p}\left(\mathbb{R}^{n}\right)}=\left\{\sum_{k=0}^{\infty} 2^{k \alpha p}\left\|f \tilde{\chi}_{k}\right\|_{L^{q(\cdot)}\left(\mathbb{R}^{n}\right)}^{p}\right\}^{1 / p}<\infty .
$$

\section{Boundedness of the Parametric Marcinkiewicz Integral Operator}

In this section we will prove the boundedness of the parametric Marcinkiewicz integral operators $\mu_{\Omega}^{\rho}$ on Herz spaces with variable exponent.

A nonnegative locally integrable function $\omega$ on $\mathbb{R}^{n}$ is said to belong to $A_{p}(1<p<\infty)$, if

$$
\begin{aligned}
& \sup _{Q}\left(\frac{1}{|Q|} \int_{Q} \omega(x) d x\right)\left(\frac{1}{|Q|} \int_{Q} \omega(x)^{1-p^{\prime}} d x\right)^{p-1} \\
& \quad<\infty
\end{aligned}
$$

where $p^{\prime}=p /(p-1), Q$ denotes a cube in $\mathbb{R}^{n}$ with its sides parallel to the coordinate axes, and $|Q|$ denotes the Lebesgue measure of $Q$.

The weighted $\left(L^{p}, L^{p}\right)$ boundedness of $\mu_{\Omega}^{\rho}$ has been proved by Shi and Jiang [12].

Lemma 6 (see [12]). Suppose that $\Omega \in L^{s}\left(S^{n-1}\right)(s>1)$ satisfying (1). If $\omega \in A_{p / s^{\prime}}, s^{\prime}<p<\infty$, then for any $f \in$ $L^{p}(\omega)$, there is a constant $C$, independent of $f$, such that

$$
\int_{\mathbb{R}^{n}}\left|\mu_{\Omega}^{\rho}(f)(x)\right|^{p} \omega(x) d x \leqslant C \int_{\mathbb{R}^{n}}|f(x)|^{p} \omega(x) d x .
$$

Lemma 7 (see [4]). Given a family $\mathscr{F}$ and an open set $E \subset \mathbb{R}^{n}$, assume that for some $p_{0}, 0<p_{0}<\infty$ and for every $\omega \in A_{\infty}$,

$$
\int_{E} f(x)^{p_{0}} \omega(x) d x \leqslant C_{0} \int_{E} g(x)^{p_{0}} \omega(x) d x,
$$

$$
(f, g) \in \mathscr{F} \text {. }
$$

Given $p(\cdot) \in \mathscr{P}^{0}(E)$ such that $p(\cdot)$ satisfies (13) and (14) in Lemma 1. Then for all $(f, g) \in \mathscr{F}$ such that $f \in L^{p(\cdot)}(E)$,

$$
\|f\|_{L^{p(\cdot)}(E)} \leqslant C\|g\|_{L^{p(\cdot)}(E)} .
$$

Since $A_{p / s^{\prime}} \subset A_{\infty}$, by Lemmas 6 and 7 it is easy to get the $\left(L^{p(\cdot)}\left(\mathbb{R}^{n}\right), L^{p(\cdot)}\left(\mathbb{R}^{n}\right)\right)$-boundedness of the parametric Marcinkiewicz integral operators $\mu_{\Omega}^{\rho}$.

To obtain the Theorem 11, we need the following lemmas.
Lemma 8 (see [13]). If $a>0,1 \leqslant s \leqslant \infty, 0<d \leqslant s$ and $-n+(n-1) d / s<v<\infty$, then

$$
\begin{aligned}
& \left(\int_{|y| \leqslant a|x|}|y|^{\nu}|\Omega(x-y)|^{d} d y\right)^{1 / d} \\
& \quad \leqslant C|x|^{(\nu+n) / d}\|\Omega\|_{L^{s}\left(S^{n-1}\right)} .
\end{aligned}
$$

Lemma 9 (see [14]). Define a variable exponent $\widetilde{q}(\cdot)$ by $1 / p(x)=1 / \widetilde{q}(x)+1 / q\left(x \in \mathbb{R}^{n}\right)$. Then we have

$$
\|f g\|_{L^{p(\cdot)}\left(\mathbb{R}^{n}\right)} \leqslant C\|f\|_{L^{\tilde{q}(\cdot)}\left(\mathbb{R}^{n}\right)}\|g\|_{L^{q}\left(\mathbb{R}^{n}\right)}
$$

for all measurable functions $f$ and $g$.

Lemma 10 (see [5]). Let $p(\cdot) \in \mathscr{P}\left(\mathbb{R}^{n}\right)$ satisfy conditions (13) and (14) in Lemma 1. Then

$$
\left\|\chi_{Q}\right\|_{L^{p(\cdot)}\left(\mathbb{R}^{n}\right)} \approx \begin{cases}|Q|^{1 / p(x)} & \text { if }|Q| \leqslant 2^{n} \text { and } x \in Q \\ |Q|^{1 / p(\infty)} & \text { if }|Q| \geqslant 1\end{cases}
$$

for every cube (or ball) $Q \subset \mathbb{R}^{n}$, where $p(\infty)=\lim _{x \rightarrow \infty} p(x)$.

Theorem 11. Suppose that $0<v \leqslant 1,0<p \leqslant \infty$, $q(\cdot) \in \mathscr{P}\left(\mathbb{R}^{n}\right)$ satisfies conditions (13) and (14) in Lemma 1; $\Omega \in L^{s}\left(\mathrm{~S}^{n-1}\right)\left(s>q^{\prime-}\right)$ and $-n \delta_{1}-v-n / s<\alpha<n \delta_{2}-v-n / s$. Then $\mu_{\Omega}^{\rho}$ is bounded on $\dot{K}_{q(\cdot)}^{\alpha, p}\left(\mathbb{R}^{n}\right)$ and $K_{q(\cdot)}^{\alpha, p}\left(\mathbb{R}^{n}\right)$.

Proof. We only prove homogeneous case. The nonhomogeneous case can be proved in the same way. We suppose $0<$ $p<\infty$, since the proof of the case $p=\infty$ is easier. Let $f \in \dot{K}_{q(\cdot)}^{\alpha, p}\left(\mathbb{R}^{n}\right)$. Denote $f_{j}=f \chi_{j}$ for each $j \in \mathbb{Z}$, then we have $f(x)=\sum_{j=-\infty}^{\infty} f_{j}(x)$. Then we have

$$
\begin{aligned}
& \left\|\mu_{\Omega}^{\rho}(f)\right\|_{\dot{K}_{q(\cdot)}^{\alpha, p}\left(\mathbb{R}^{n}\right)}=\left\{\sum_{k=-\infty}^{\infty} 2^{k \alpha p}\left\|\mu_{\Omega}^{\rho}(f) \chi_{k}\right\|_{L^{q(\cdot)}\left(\mathbb{R}^{n}\right)}^{p}\right\}^{1 / p} \\
& \leqslant C\left\{\sum_{k=-\infty}^{\infty} 2^{k \alpha p}\right. \\
& \left..\left(\sum_{j=-\infty}^{k-2}\left\|\mu_{\Omega}^{\rho}\left(f_{j}\right) \chi_{k}\right\|_{L^{q(\cdot)}\left(\mathbb{R}^{n}\right)}\right)^{p}\right\}^{1 / p} \\
& +C\left\{\sum_{k=-\infty}^{\infty} 2^{k \alpha p}\left(\sum_{j=k-1}^{k+1}\left\|\mu_{\Omega}^{\rho}\left(f_{j}\right) \chi_{k}\right\|_{L^{q(\cdot)}\left(\mathbb{R}^{n}\right)}\right)^{p}\right\}^{1 / p} \\
& +C\left\{\sum_{k=-\infty}^{\infty} 2^{k \alpha p}\left(\sum_{j=k+2}^{\infty}\left\|\mu_{\Omega}^{\rho}\left(f_{j}\right) \chi_{k}\right\|_{L^{q(\cdot)}\left(\mathbb{R}^{n}\right)}\right)^{p}\right\}^{1 / p} \\
& \quad=C I_{1}+C I_{2}+C I_{3} .
\end{aligned}
$$

We first estimate $I_{2}$, by the $\left(L^{q(\cdot)}\left(\mathbb{R}^{n}\right), L^{q(\cdot)}\left(\mathbb{R}^{n}\right)\right)$-boundedness of the commutator $\mu_{\Omega}^{\rho}$; we have

$$
I_{2} \leqslant C\left\{\sum_{k=-\infty}^{\infty} 2^{k \alpha p}\left\|f_{k}\right\|_{L^{q(\cdot)}}^{p}\right\}^{1 / p}=C\|f\|_{\dot{K}_{q(\cdot)}^{\alpha, p}\left(\mathbb{R}^{n}\right)} .
$$


Now we estimate $I_{1}$. We consider

$$
\begin{aligned}
& \left|\mu_{\Omega}^{\rho}\left(f_{j}\right)(x)\right| \\
& \leqslant\left(\int_{0}^{|x|}\left|\int_{|x-y| \leqslant t} \frac{\Omega(x-y)}{|x-y|^{n-\rho}} f_{j}(y) d y\right|^{2} \frac{d t}{t^{2 \rho+1}}\right)^{1 / 2} \\
& \quad+\left(\int_{|x|}^{\infty}\left|\int_{|x-y| \leqslant t} \frac{\Omega(x-y)}{|x-y|^{n-\rho}} f_{j}(y) d y\right|^{2} \frac{d t}{t^{2 \rho+1}}\right)^{1 / 2} \\
& =: I_{11}+I_{12} .
\end{aligned}
$$

Note that $x \in A_{k}, y \in A_{j}$, and $j \leqslant k-2$. So we know that $|x-y| \sim|x|$, and by mean value theorem we have

$$
\left|\frac{1}{|x-y|^{2 \rho}}-\frac{1}{|x|^{2 \rho}}\right| \leqslant \frac{|y|}{|x-y|^{2 \rho+1}} .
$$

By (31), the Minkowski inequality, and the generalized Hölder inequality we have

$$
\begin{aligned}
I_{11} & \leqslant C \int_{\mathbb{R}^{n}} \frac{|\Omega(x-y)|}{|x-y|^{n-\rho}}\left|f_{j}(y)\right|\left(\int_{|x-y|}^{|x|} \frac{d t}{t^{2 \rho+1}}\right)^{1 / 2} d y \\
& \leqslant C \int_{\mathbb{R}^{n}} \frac{|\Omega(x-y)|}{|x-y|^{n-\rho}}\left|f_{j}(y)\right| \\
& \cdot\left|\frac{1}{|x-y|^{2 \rho}}-\frac{1}{|x|^{2 \rho}}\right|^{1 / 2} d y \\
& \leqslant C \int_{\mathbb{R}^{n}} \frac{|\Omega(x-y)|}{|x-y|^{n-\rho}}\left|f_{j}(y)\right| \frac{|y|^{1 / 2}}{|x-y|^{\rho+1 / 2}} d y \leqslant C \\
& \cdot \frac{2^{j / 2}}{|x|^{n+1 / 2}} \int_{A_{j}}|\Omega(x-y)||f(y)| d y \\
& \leqslant C 2^{(j-k) / 2} 2^{-k n}\left\|f_{j}\right\|_{L^{q(\cdot)}\left(\mathbb{R}^{n}\right)}\left\|\Omega(x-\cdot) \chi_{j}(\cdot)\right\|_{L^{q^{\prime}(\cdot)}\left(\mathbb{R}^{n}\right)}
\end{aligned}
$$

Similarly, we consider $I_{12}$. Noting that $|x-y| \sim|x|$, by the Minkowski inequality and the generalized Hölder inequality, we have

$$
\begin{aligned}
I_{12} & \leqslant C \int_{\mathbb{R}^{n}} \frac{|\Omega(x-y)|}{|x-y|^{n-\rho}}\left|f_{j}(y)\right|\left(\int_{|x|}^{\infty} \frac{d t}{t^{2 \rho+1}}\right)^{1 / 2} d y \\
& \leqslant C \int_{\mathbb{R}^{n}} \frac{|\Omega(x-y)|}{|x-y|^{n}}\left|f_{j}(y)\right| d y \\
& \leqslant C 2^{-k n}\left\|f_{j}\right\|_{L^{q(\cdot)}\left(\mathbb{R}^{n}\right)}\left\|\Omega(x-\cdot) \chi_{j}(\cdot)\right\|_{L^{q^{\prime}(\cdot)\left(\mathbb{R}^{n}\right)}} \cdot
\end{aligned}
$$

So we have

$$
\begin{aligned}
& \left|\mu_{\Omega}^{\rho}\left(f_{j}\right)(x)\right| \\
& \quad \leqslant C 2^{-k n}\left\|f_{j}\right\|_{L^{q(\cdot)}\left(\mathbb{R}^{n}\right)}\left\|\Omega(x-\cdot) \chi_{j}(\cdot)\right\|_{L^{q^{(\cdot)}\left(\mathbb{R}^{n}\right)}} .
\end{aligned}
$$

Noting $s>q^{\prime-}$, we denote $\widetilde{q^{\prime}}(\cdot)>1$ and $1 / q^{\prime}(x)=1 / \widetilde{q^{\prime}}(x)+$ $1 / s$. By Lemmas 8 and 9 we have

$$
\begin{aligned}
& \left\|\Omega(x-\cdot) \chi_{j}(\cdot)\right\|_{L^{q^{\prime}(\cdot)\left(\mathbb{R}^{n}\right)}} \\
& \leqslant\left\|\Omega(x-\cdot) \chi_{j}(\cdot)\right\|_{L^{s}\left(\mathbb{R}^{n}\right)}\left\|\chi_{j}(\cdot)\right\|_{L^{q^{T^{\prime} \cdot(\cdot)}\left(\mathbb{R}^{n}\right)}} \\
& \leqslant\left\|\Omega(x-\cdot) \chi_{j}(\cdot)\right\|_{L^{s}\left(\mathbb{R}^{n}\right)}\left\|\chi_{B_{j}}\right\|_{L^{q^{\top}(\cdot)}\left(\mathbb{R}^{n}\right)} \\
& \leqslant C 2^{-j v}\left(\int_{A_{j}}|\Omega(x-y)|^{s}|y|^{s v} d y\right)^{1 / s}\left\|\chi_{B_{j}}\right\|_{L^{q^{\top}(\cdot)\left(\mathbb{R}^{n}\right)}} \\
& \quad \leqslant C 2^{-j v} 2^{k(v+n / s)}\|\Omega\|_{L^{s}\left(S^{n-1}\right)}\left\|\chi_{B_{j}}\right\|_{L^{q^{\top}(\cdot)}\left(\mathbb{R}^{n}\right)} \cdot
\end{aligned}
$$

When $\left|B_{j}\right| \leqslant 2^{n}$ and $x_{j} \in B_{j}$, by Lemma 10 we have

$$
\left\|\chi_{B_{j}}\right\|_{L^{q^{\prime}(\cdot)\left(\mathbb{R}^{n}\right)}} \approx\left|B_{j}\right|^{1 / \widetilde{q^{\prime}}\left(x_{j}\right)} \approx\left\|\chi_{B_{j}}\right\|_{L^{q^{\prime}(\cdot)\left(\mathbb{R}^{n}\right)}}\left|B_{j}\right|^{-1 / s} .
$$

When $\left|B_{j}\right| \geqslant 1$ we have

$$
\left\|\chi_{B_{j}}\right\|_{L^{\tilde{q}^{\prime}(\cdot)\left(\mathbb{R}^{n}\right)}} \approx\left|B_{j}\right|^{1 / \widetilde{q^{\prime}}(\infty)} \approx\left\|\chi_{B_{j}}\right\|_{L^{q^{\prime}(\cdot)}\left(\mathbb{R}^{n}\right)}\left|B_{j}\right|^{-1 / s} .
$$

So we obtain $\left\|\chi_{B_{j}}\right\|_{L^{q^{\prime}(\cdot)\left(\mathbb{R}^{n}\right)}} \approx\left\|\chi_{B_{j}}\right\|_{L^{q^{\prime}(\cdot)\left(\mathbb{R}^{n}\right)}}\left|B_{j}\right|^{-1 / s}$.

By Lemmas 3 and 4 we have

$$
\begin{aligned}
& \left\|\mu_{\Omega}^{\rho}\left(f_{j}\right) \chi_{k}\right\|_{L^{q(\cdot)}\left(\mathbb{R}^{n}\right)} \leqslant C 2^{-k n}\left\|f_{j}\right\|_{L^{q(\cdot)}\left(\mathbb{R}^{n}\right)} \\
& \cdot\left\|\Omega(x-\cdot) \chi_{j}(\cdot)\right\|_{L^{q^{\prime}(\cdot)}\left(\mathbb{R}^{n}\right)}\left\|\chi_{k}\right\|_{L^{q(\cdot)}\left(\mathbb{R}^{n}\right)} \\
& \leqslant C 2^{-k n} 2^{-j v} 2^{k(v+n / s)}\|\Omega\|_{L^{s}\left(S^{n-1}\right)}\left\|f_{j}\right\|_{L^{q(\cdot)}\left(\mathbb{R}^{n}\right)} \\
& \cdot\left\|\chi_{B_{j}}\right\|_{L^{q^{q^{(} \cdot(\cdot)}\left(\mathbb{R}^{n}\right)}}\left\|\chi_{B_{k}}\right\|_{L^{q(\cdot)}\left(\mathbb{R}^{n}\right)} \\
& \leqslant C 2^{-k n} 2^{-j v} 2^{k(v+n / s)}\|\Omega\|_{L^{s}\left(S^{n-1}\right)}\left\|f_{j}\right\|_{L^{q(\cdot)}\left(\mathbb{R}^{n}\right)} \\
& \times\left\|\chi_{B_{j}}\right\|_{L^{q^{\prime}(\cdot)}\left(\mathbb{R}^{n}\right)}\left|B_{j}\right|^{-1 / s}\left\|\chi_{B_{k}}\right\|_{L^{q(\cdot)}\left(\mathbb{R}^{n}\right)} \\
& \leqslant C 2^{(k-j)(v+n / s)}\|\Omega\|_{L^{s}\left(S^{n-1}\right)}\left\|f_{j}\right\|_{L^{q(\cdot)}\left(\mathbb{R}^{n}\right)} \frac{\left\|\chi_{B_{j}}\right\|_{L^{q^{(\cdot)}\left(\mathbb{R}^{n}\right)}}}{\left\|\chi_{B_{k}}\right\|_{L^{q^{(\cdot)}\left(\mathbb{R}^{n}\right)}}} \\
& \leqslant C 2^{(j-k)\left(n \delta_{2}-\nu-n / s\right)}\|\Omega\|_{L^{s}\left(S^{n-1}\right)}\left\|f_{j}\right\|_{L^{q(\cdot)}\left(\mathbb{R}^{n}\right)} .
\end{aligned}
$$

Thus we obtain

$$
\begin{gathered}
I_{1} \leqslant C\|\Omega\|_{L^{s}\left(S^{n-1}\right)}\left\{\sum _ { k = - \infty } ^ { \infty } 2 ^ { k \alpha p } \left(\sum_{j=-\infty}^{k-2} 2^{(j-k)\left(n \delta_{2}-\gamma-n / s\right)}\right.\right. \\
\left.\left.\cdot\left\|f_{j}\right\|_{L^{q(\cdot)}\left(\mathbb{R}^{n}\right)}\right)^{p}\right\}^{1 / p}=C\|\Omega\|_{L^{s}\left(S^{n-1}\right)}
\end{gathered}
$$




$$
\begin{aligned}
& \left\{\sum _ { k = - \infty } ^ { \infty } \left(\sum_{j=-\infty}^{k-2} 2^{j \alpha} 2^{(j-k)\left(n \delta_{2}-v-n / s-\alpha\right)}\right.\right. \\
& \left.\left.\cdot\left\|f_{j}\right\|_{L^{q(\cdot)}\left(\mathbb{R}^{n}\right)}\right)^{p}\right\}^{1 / p} .
\end{aligned}
$$

If $1<p<\infty$, take $1 / p+1 / p^{\prime}=1$. Since $n \delta_{2}-v-n / s-\alpha>0$, by the Hölder inequality we have

$$
\begin{aligned}
& I_{1} \leqslant C\|\Omega\|_{L^{s}\left(S^{n-1}\right)}\left\{\sum _ { k = - \infty } ^ { \infty } \left(\sum_{j=-\infty}^{k-2} 2^{j \alpha p}\left\|f_{j}\right\|_{L^{q(\cdot)}\left(\mathbb{R}^{n}\right)}^{p}\right.\right. \\
& \left.\cdot 2^{(j-k)\left(n \delta_{2}-v-n / s-\alpha\right) p / 2}\right) \\
& \left.\times\left(\sum_{j=-\infty}^{k-2} 2^{(j-k)\left(n \delta_{2}-\nu-n / s-\alpha\right) p^{\prime} / 2}\right)^{p / p^{\prime}}\right\}^{1 / p} \\
& \leqslant C\|\Omega\|_{L^{s}\left(S^{n-1}\right)}\left\{\sum _ { k = - \infty } ^ { \infty } \left(\sum_{j=-\infty}^{k-2} 2^{j \alpha p}\left\|f_{j}\right\|_{L^{q(\cdot)}\left(\mathbb{R}^{n}\right)}^{p}\right.\right. \\
& \left.\left.\times 2^{(j-k)\left(n \delta_{2}-\nu-n / s-\alpha\right) p / 2}\right)\right\}^{1 / p}=C\|\Omega\|_{L^{s}\left(S^{n-1}\right)} \\
& \left\{\sum_{j=-\infty}^{\infty} 2^{j \alpha p}\left\|f_{j}\right\|_{L^{q(\cdot)}\left(\mathbb{R}^{n}\right)}^{p}\right. \\
& \left.\times\left(\sum_{k=j+2}^{\infty} 2^{(j-k)\left(n \delta_{2}-\nu-n / s-\alpha\right) p / 2}\right)\right\}^{1 / p} \\
& \leqslant C\left\{\sum_{j=-\infty}^{\infty} 2^{j \alpha p}\left\|f_{j}\right\|_{L^{q(\cdot)}\left(\mathbb{R}^{n}\right)}^{p}\right\}^{1 / p}=C\|f\|_{\dot{K}_{q(\cdot)}^{\alpha, p}\left(\mathbb{R}^{n}\right)} .
\end{aligned}
$$

If $0<p \leqslant 1$, then we have

$$
\begin{aligned}
I_{1} \leqslant & C\|\Omega\|_{L^{s}\left(S^{n-1}\right)}\left\{\sum_{k=-\infty}^{\infty} \sum_{j=-\infty}^{k-2} 2^{j \alpha p}\right. \\
& \left.\cdot 2^{(j-k)\left(n \delta_{2}-v-n / s-\alpha\right) p}\left\|f_{j}\right\|_{L^{q(\cdot)}\left(\mathbb{R}^{n}\right)}^{p}\right\} \\
& =C\|\Omega\|_{L^{s}\left(S^{n-1}\right)}\left\{\sum_{j=-\infty}^{\infty} 2^{j \alpha p}\left\|f_{j}\right\|_{L^{q(\cdot)}\left(\mathbb{R}^{n}\right)}^{p}\right. \\
& \left.\cdot\left(\sum_{k=j+2}^{\infty} 2^{(j-k)\left(n \delta_{2}-\nu-n / s-\alpha\right) p}\right)\right\}^{1 / p} \leqslant C\|f\|_{\dot{K}_{q(\cdot)}^{\alpha, p}\left(\mathbb{R}^{n}\right)} .
\end{aligned}
$$

Let us now estimate $I_{3}$. Note that $x \in A_{k}, y \in A_{j}$, and $j \geqslant k+2$, so we have $|x-y| \sim|y|$. We consider

$$
\begin{aligned}
& \left|\mu_{\Omega}^{\rho}\left(f_{j}\right)(x)\right| \\
& \leqslant\left(\int_{0}^{|y|}\left|\int_{|x-y| \leqslant t} \frac{\Omega(x-y)}{|x-y|^{n-\rho}} f_{j}(y) d y\right|^{2} \frac{d t}{t^{2 \rho+1}}\right)^{1 / 2} \\
& \quad+\left(\int_{|y|}^{\infty}\left|\int_{|x-y| \leqslant t} \frac{\Omega(x-y)}{|x-y|^{n-\rho}} f_{j}(y) d y\right|^{2} \frac{d t}{t^{2 \rho+1}}\right)^{1 / 2} \\
& =: I_{31}+I_{32} .
\end{aligned}
$$

Similar to the estimate for $I_{11}$, we get

$$
\begin{aligned}
& I_{31} \\
& \quad \leqslant C 2^{(k-j) / 2} 2^{-j n}\left\|f_{j}\right\|_{L^{q(\cdot)}\left(\mathbb{R}^{n}\right)}\left\|\Omega(x-\cdot) \chi_{j}(\cdot)\right\|_{L^{q^{\prime}(\cdot)}\left(\mathbb{R}^{n}\right)} .
\end{aligned}
$$

Similar to the estimate for $I_{12}$, we get

$$
I_{32} \leqslant C 2^{-j n}\left\|f_{j}\right\|_{L^{q(\cdot)}\left(\mathbb{R}^{n}\right)}\left\|\Omega(x-\cdot) \chi_{j}(\cdot)\right\|_{L^{q^{(\cdot)}\left(\mathbb{R}^{n}\right)}} .
$$

So we have

$$
\begin{aligned}
& \left|\mu_{\Omega}^{\rho}\left(f_{j}\right)(x)\right| \\
& \quad \leqslant C 2^{-j n}\left\|f_{j}\right\|_{L^{q(\cdot)}\left(\mathbb{R}^{n}\right)}\left\|\Omega(x-\cdot) \chi_{j}(\cdot)\right\|_{L^{q^{\prime}(\cdot)}\left(\mathbb{R}^{n}\right)} .
\end{aligned}
$$

By Lemmas 3 and 4 we have

$$
\begin{aligned}
& \left\|\mu_{\Omega}^{\rho}\left(f_{j}\right) \chi_{k}\right\|_{L^{q(\cdot)}\left(\mathbb{R}^{n}\right)} \leqslant C 2^{-j n}\left\|f_{j}\right\|_{L^{q(\cdot)}\left(\mathbb{R}^{n}\right)} \\
& \cdot\left\|\Omega(x-\cdot) \chi_{j}(\cdot)\right\|_{L^{q^{\prime}(\cdot)\left(\mathbb{R}^{n}\right)}}\left\|\chi_{k}\right\|_{L^{q^{(\cdot)}\left(\mathbb{R}^{n}\right)}} \\
& \leqslant C 2^{-j n} 2^{-j v} 2^{k(\gamma+n / s)}\|\Omega\|_{L^{s}\left(S^{n-1}\right)}\left\|f_{j}\right\|_{L^{q(\cdot)}\left(\mathbb{R}^{n}\right)} \\
& \cdot\left\|\chi_{B_{j}}\right\|_{L^{q^{\top} \cdot(\cdot)\left(\mathbb{R}^{n}\right)}}\left\|\chi_{B_{k}}\right\|_{L^{q(\cdot)}\left(\mathbb{R}^{n}\right)} \\
& \leqslant C 2^{-j n} 2^{-j v} 2^{k(v+n / s)}\|\Omega\|_{L^{s}\left(S^{n-1}\right)}\left\|f_{j}\right\|_{L^{q^{(\cdot)}\left(\mathbb{R}^{n}\right)}} \\
& \times\left\|\chi_{B_{j}}\right\|_{L^{q^{\prime}(\cdot)\left(\mathbb{R}^{n}\right)}}\left|B_{j}\right|^{-1 / s}\left\|\chi_{B_{k}}\right\|_{L^{q(\cdot)}\left(\mathbb{R}^{n}\right)} \\
& \leqslant C 2^{(k-j)(v+n / s)}\|\Omega\|_{L^{s}\left(S^{n-1}\right)}\left\|f_{j}\right\|_{L^{q(\cdot)}\left(\mathbb{R}^{n}\right)} \frac{\left\|\chi_{B_{k}}\right\|_{L^{q \cdot(\cdot)}\left(\mathbb{R}^{n}\right)}}{\left\|\chi_{B_{j}}\right\|_{L^{q(\cdot)}\left(\mathbb{R}^{n}\right)}} \\
& \leqslant C 2^{(k-j)\left(n \delta_{1}+v+n / s\right)}\|\Omega\|_{L^{s}\left(S^{n-1}\right)}\left\|f_{j}\right\|_{L^{q(\cdot)}\left(\mathbb{R}^{n}\right)} .
\end{aligned}
$$

Thus we obtain

$$
\begin{gathered}
I_{3} \leqslant C\|\Omega\|_{L^{s}\left(S^{n-1}\right)}\left\{\sum _ { k = - \infty } ^ { \infty } 2 ^ { k \alpha p } \left(\sum_{j=k+2}^{\infty} 2^{(k-j)\left(n \delta_{1}+\nu+n / s\right)}\right.\right. \\
\left.\left.\cdot\left\|f_{j}\right\|_{L^{q(\cdot)}\left(\mathbb{R}^{n}\right)}\right)^{p}\right\}^{1 / p}=C\|\Omega\|_{L^{s}\left(S^{n-1}\right)}
\end{gathered}
$$




$$
\begin{aligned}
& \left\{\sum _ { k = - \infty } ^ { \infty } \left(\sum_{j=k+2}^{\infty} 2^{j \alpha}\right.\right. \\
& \left.\left.\cdot 2^{(k-j)\left(n \delta_{1}+v+n / s+\alpha\right)}\left\|f_{j}\right\|_{L^{q(\cdot)}\left(\mathbb{R}^{n}\right)}\right)^{p}\right\}^{1 / p} .
\end{aligned}
$$

If $1<p<\infty$, take $1 / p+1 / p^{\prime}=1$. Since $n \delta_{1}+v+n / s+\alpha>0$, by the Hölder inequality we have

$$
\begin{aligned}
& I_{3} \leqslant C\|\Omega\|_{L^{s}\left(S^{n-1}\right)}\left\{\sum _ { k = - \infty } ^ { \infty } \left(\sum_{j=k+2}^{\infty} 2^{j \alpha p}\left\|f_{j}\right\|_{L^{q(\cdot)}\left(\mathbb{R}^{n}\right)}^{p}\right.\right. \\
& \left.\cdot 2^{(k-j)\left(n \delta_{1}+v+n / s+\alpha\right) p / 2}\right) \\
& \left.\times\left(\sum_{j=k+2}^{\infty} 2^{(k-j)\left(n \delta_{1}+\nu+n / s+\alpha\right) p^{\prime} / 2}\right)^{p / p^{\prime}}\right\}^{1 / p} \\
& \leqslant C\|\Omega\|_{L^{s}\left(S^{n-1}\right)}\left\{\sum _ { k = - \infty } ^ { \infty } \left(\sum_{j=k+2}^{\infty} 2^{j \alpha p}\left\|f_{j}\right\|_{L^{q(\cdot)}\left(\mathbb{R}^{n}\right)}^{p}\right.\right. \\
& \left.\left.\times 2^{(k-j)\left(n \delta_{1}+\nu+n / s+\alpha\right) p / 2}\right)\right\}^{1 / p}=C\|\Omega\|_{L^{s}\left(S^{n-1}\right)} \\
& \left\{\sum_{j=-\infty}^{\infty} 2^{j \alpha p}\left\|f_{j}\right\|_{L^{q(\cdot)}\left(\mathbb{R}^{n}\right)}^{p}\right. \\
& \left.\times\left(\sum_{k=-\infty}^{j-2} 2^{(k-j)\left(n \delta_{1}+\nu+n / s+\alpha\right) p / 2}\right)\right\}^{1 / p} \\
& \leqslant C\left\{\sum_{j=-\infty}^{\infty} 2^{j \alpha p}\left\|f_{j}\right\|_{L^{q(\cdot)}\left(\mathbb{R}^{n}\right)}^{p}\right\}^{1 / p}=C\|f\|_{\dot{K}_{q(\cdot)}^{\alpha, p}\left(\mathbb{R}^{n}\right)} .
\end{aligned}
$$

If $0<p \leqslant 1$, then we have

$$
\begin{aligned}
I_{3} \leqslant & C\|\Omega\|_{L^{s}\left(S^{n-1}\right)}\left\{\sum_{k=-\infty}^{\infty} \sum_{j=k+2}^{\infty} 2^{j \alpha p} 2^{(k-j)\left(n \delta_{1}+\nu+n / s+\alpha\right) p}\right. \\
& \left.\cdot\left\|f_{j}\right\|_{L^{q(\cdot)}\left(\mathbb{R}^{n}\right)}^{p}\right\}^{1 / p}=C\|\Omega\|_{L^{s}\left(S^{n-1}\right)} \\
& \cdot\left\{\sum_{j=-\infty}^{\infty} 2^{j \alpha p}\left\|f_{j}\right\|_{L^{q(\cdot)}\left(\mathbb{R}^{n}\right)}^{p}\right. \\
& \left.\cdot\left(\sum_{k=-\infty}^{j-2} 2^{(k-j)\left(n \delta_{1}+\nu+n / s+\alpha\right) p}\right)\right\}^{1 / p} \leqslant C\|f\|_{\dot{K}_{q(\cdot)}^{\alpha, p}\left(\mathbb{R}^{n}\right)} .
\end{aligned}
$$

Thus by (28), (29) and (40), (41), (48), and (49) we complete the proof of Theorem 11.

\section{BMO Estimate for the Higher-Order Commutator of Parametric Marcinkiewicz Integral Operator}

Let us first recall that the space $\operatorname{BMO}\left(\mathbb{R}^{n}\right)$ consists of all locally integrable functions $f$ such that

$$
\|f\|_{*}=\sup _{\mathrm{Q}} \frac{1}{|Q|} \int_{\mathrm{Q}}\left|f(x)-f_{\mathrm{Q}}\right| d x<\infty,
$$

where $f_{\mathrm{Q}}=|Q|^{-1} \int_{\mathrm{Q}} f(y) d y$, the supremum is taken over all cubes $Q \subset \mathbb{R}^{n}$ with sides parallel to the coordinate axes, and $|Q|$ denotes the Lebesgue measure of $Q$.

Let $b \in \operatorname{BMO}\left(\mathbb{R}^{n}\right)$. The weighted $\left(L^{p}, L^{p}\right)$ boundedness of $\left[b^{m}, \mu_{\Omega}^{\rho}\right]$ has been proved by Shi and Jiang [12].

Lemma 12 (see [12]). Suppose that $\Omega \in L^{s}\left(S^{n-1}\right)(s>1)$ satisfying (1). If $b(x) \in \mathrm{BMO}\left(\mathbb{R}^{n}\right)$ and $\omega \in A_{p / s^{\prime}}, s^{\prime}<p<\infty$, then for any $f \in L^{p}(\omega)$, there is a constant $C$, independent of $f$, such that

$$
\begin{gathered}
\int_{\mathbb{R}^{n}}\left|\left[b^{m}, \mu_{\Omega}^{\rho}\right](f)(x)\right|^{p} \omega(x) d x \\
\leqslant C \int_{\mathbb{R}^{n}}|f(x)|^{p} \omega(x) d x .
\end{gathered}
$$

By Lemmas 12 and 7 it is easy to get the $\left(L^{p(\cdot)}\left(\mathbb{R}^{n}\right)\right.$, $\left.L^{p(\cdot)}\left(\mathbb{R}^{n}\right)\right)$-boundedness of the commutator $\left[b^{m}, \mu_{\Omega}^{\rho}\right]$.

Next, we will give the corresponding result about the commutator $\left[b^{m}, \mu_{\Omega}^{\rho}\right]$ on Herz spaces with variable exponent.

Theorem 13. Suppose that $b \in \mathrm{BMO}\left(\mathbb{R}^{n}\right), 0<v \leqslant 1,0<p \leqslant$ $\infty, q(\cdot) \in \mathscr{P}\left(\mathbb{R}^{n}\right)$ satisfies conditions (13) and (14) in Lemma 1; $\Omega \in L^{s}\left(\mathrm{~S}^{n-1}\right)\left(s>q^{\prime-}\right)$ and $-n \delta_{1}-v-n / s<\alpha<n \delta_{2}-v-n / s$. Then $\left[b^{m}, \mu_{\Omega}^{\rho}\right]$ is bounded on $\dot{K}_{q(\cdot)}^{\alpha, p}\left(\mathbb{R}^{n}\right)$ and $K_{q(\cdot)}^{\alpha, p}\left(\mathbb{R}^{n}\right)$.

In the proof of Theorem 13, we also need the following lemma.

Lemma 14 (see [15]). Let $p(\cdot) \in \mathscr{B}\left(\mathbb{R}^{n}\right), m$ be a positive integer, and $B$ be a ball in $\mathbb{R}^{n}$. Then we have that, for all $b \in \operatorname{BMO}\left(\mathbb{R}^{n}\right)$ and all $j, i \in \mathbb{Z}$ with $j>i$,

$$
\begin{aligned}
& \frac{1}{C}\|b\|_{*}^{m} \leqslant \sup _{B} \frac{1}{\left\|\chi_{B}\right\|_{L^{p(\cdot)}\left(\mathbb{R}^{n}\right)}}\left\|\left(b-b_{B}\right)^{m} \chi_{B}\right\|_{L^{p(\cdot)}\left(\mathbb{R}^{n}\right)} \\
& \quad \leqslant C\|b\|_{*}^{m}, \\
& \left\|\left(b-b_{B_{i}}\right)^{m} \chi_{B_{j}}\right\|_{L^{p(\cdot)}\left(\mathbb{R}^{n}\right)} \\
& \quad \leqslant C(j-i)^{m}\|b\|_{*}^{m}\left\|\chi_{B_{j}}\right\|_{L^{p(\cdot)}\left(\mathbb{R}^{n}\right)},
\end{aligned}
$$

where $B_{i}=\left\{x \in \mathbb{R}^{n}:|x| \leqslant 2^{i}\right\}$ and $B_{j}=\left\{x \in \mathbb{R}^{n}:|x| \leqslant 2^{j}\right\}$. 
Proof of Theorem 13. Similar to Theorem 11, we only prove homogeneous case and still suppose $0<p<\infty$. Let $f \in$ $\dot{K}_{q(\cdot)}^{\alpha, p}\left(\mathbb{R}^{n}\right)$. Denote $f_{j}=f \chi_{j}$ for each $j \in \mathbb{Z}$, then we have $f(x)=\sum_{j=-\infty}^{\infty} f_{j}(x)$. Then

$$
\begin{aligned}
& \left\|\left[b^{m}, \mu_{\Omega}^{\rho}\right](f)\right\|_{\dot{K}_{q(\cdot)}^{\alpha, p}\left(\mathbb{R}^{n}\right)}=\left\{\sum_{k=-\infty}^{\infty} 2^{k \alpha p}\right. \\
& \left.\cdot\left\|\left[b^{m}, \mu_{\Omega}^{\rho}\right](f) \chi_{k}\right\|_{L^{q(\cdot)}\left(\mathbb{R}^{n}\right)}^{p}\right\}^{1 / p} \leqslant C\left\{\sum_{k=-\infty}^{\infty} 2^{k \alpha p}\right. \\
& \left.\cdot\left(\sum_{j=-\infty}^{k-2}\left\|\left[b^{m}, \mu_{\Omega}^{\rho}\right]\left(f_{j}\right) \chi_{k}\right\|_{L^{q(\cdot)}\left(\mathbb{R}^{n}\right)}\right)^{p}\right\}^{1 / p} \\
& +C\left\{\sum_{k=-\infty}^{\infty} 2^{k \alpha p}\right. \\
& \left.\cdot\left(\sum_{j=k-1}^{k+1}\left\|\left[b^{m}, \mu_{\Omega}^{\rho}\right]\left(f_{j}\right) \chi_{k}\right\|_{L^{q(\cdot)}\left(\mathbb{R}^{n}\right)}\right)^{p}\right\}^{1 / p}
\end{aligned}
$$

$+C\left\{\sum_{k=-\infty}^{\infty} 2^{k \alpha p}\right.$

$\left.\cdot\left(\sum_{j=k+2}^{\infty}\left\|\left[b^{m}, \mu_{\Omega}^{\rho}\right]\left(f_{j}\right) \chi_{k}\right\|_{L^{q(\cdot)}\left(\mathbb{R}^{n}\right)}\right)^{p}\right\}^{1 / p}=: C J_{1}$

$+\mathrm{CJ}_{2}+\mathrm{CJ}_{3}$.

Noting $\left[b^{m}, \mu_{\Omega}^{\rho}\right]$ is bounded on $L^{q(\cdot)}\left(\mathbb{R}^{n}\right)$, so we have

$$
J_{2} \leqslant C\left\{\sum_{k=-\infty}^{\infty} 2^{k \alpha p}\left\|f_{k}\right\|_{L^{q \cdot(\cdot)}}^{p}\right\}^{1 / p}=C\|f\|_{\mathbb{K}_{q(\cdot)}^{\alpha, p}\left(\mathbb{R}^{n}\right)} .
$$

Now we estimate $J_{1}$. We consider

$$
\begin{aligned}
\left|\left[b^{m}, \mu_{\Omega}^{\rho}\right]\left(f_{j}\right)(x)\right| \leqslant & \left(\int_{0}^{|x|}\left|\int_{|x-y| \leqslant t} \frac{\Omega(x-y)}{|x-y|^{n-\rho}}[b(x)-b(y)]^{m} f_{j}(y) d y\right|^{2} \frac{d t}{t^{2 \rho+1}}\right)^{1 / 2} \\
& +\left(\int_{|x|}^{\infty}\left|\int_{|x-y| \leqslant t} \frac{\Omega(x-y)}{|x-y|^{n-\rho}}[b(x)-b(y)]^{m} f_{j}(y) d y\right|^{2} \frac{d t}{t^{2 \rho+1}}\right)^{1 / 2}=: J_{11}+J_{12} .
\end{aligned}
$$

Note that $x \in A_{k}, y \in A_{j}$, and $j \leqslant k-2$, and we know that $|x-y| \sim|x|$. By (31), the Minkowski inequality, and the generalized Hölder inequality we have

$$
\begin{aligned}
J_{11} & \leqslant C \int_{\mathbb{R}^{n}} \frac{|\Omega(x-y)|}{|x-y|^{n-\rho}}|b(x)-b(y)|^{m}\left|f_{j}(y)\right| \\
& \cdot\left(\int_{|x-y|}^{|x|} \frac{d t}{t^{2 \rho+1}}\right)^{1 / 2} d y \\
& \leqslant C \int_{\mathbb{R}^{n}} \frac{|\Omega(x-y)|}{|x-y|^{n-\rho}}|b(x)-b(y)|^{m}\left|f_{j}(y)\right| \\
& \cdot\left|\frac{1}{|x-y|^{2 \rho}}-\frac{1}{|x|^{2 \rho}}\right|^{1 / 2} d y \\
& \leqslant C \int_{\mathbb{R}^{n}} \frac{|\Omega(x-y)||b(x)-b(y)|^{m}\left|f_{j}(y)\right|}{|x-y|^{n-\rho}} \frac{|y|^{1 / 2}}{|x-y|^{\rho+1 / 2}} d y \\
& \leqslant C \frac{2^{j / 2}}{|x|^{n+1 / 2}} \int_{A_{j}}|\Omega(x-y)||b(x)-b(y)|^{m}|f(y)| d y \\
& \leqslant C 2^{(j-k) / 2} 2^{-k n}\left\{\left|b(x)-b_{B_{j}}\right|^{m} \int_{A_{j}}|\Omega(x-y)|\left|f_{j}(y)\right| d y\right.
\end{aligned}
$$

$$
\begin{aligned}
& \left.+\int_{A_{j}}|\Omega(x-y)|\left|b_{B_{j}}-b(y)\right|^{m}\left|f_{j}(y)\right| d y\right\} \\
& \leqslant C 2^{(j-k) / 2} 2^{-k n}\left\|f_{j}\right\|_{L^{q^{(\cdot)}\left(\mathbb{R}^{n}\right)}}\left\{\left|b(x)-b_{B_{j}}\right|^{m}\right. \\
& \cdot\left\|\Omega(x-\cdot) \chi_{j}(\cdot)\right\|_{L^{q^{\prime}(\cdot)\left(\mathbb{R}^{n}\right)}} \\
& \left.+\left\|\Omega(x-\cdot)\left(b_{B_{j}}-b(\cdot)\right)^{m} \chi_{j}(\cdot)\right\|_{L^{q^{\prime}(\cdot)\left(\mathbb{R}^{n}\right)}}\right\} .
\end{aligned}
$$

Similarly, we consider $J_{12}$. Noting that $|x-y| \sim|x|$, by the Minkowski inequality and the generalized Hölder inequality, we have

$$
\begin{aligned}
J_{12} & \leqslant C \int_{\mathbb{R}^{n}} \frac{|\Omega(x-y)|}{|x-y|^{n-\rho}}|b(x)-b(y)|^{m}\left|f_{j}(y)\right| \\
& \cdot\left(\int_{|x|}^{\infty} \frac{d t}{t^{2 \rho+1}}\right)^{1 / 2} d y \\
& \leqslant C \int_{\mathbb{R}^{n}} \frac{|\Omega(x-y)|}{|x-y|^{n}}|b(x)-b(y)|^{m}\left|f_{j}(y)\right| d y
\end{aligned}
$$


8

Journal of Function Spaces

$$
\begin{aligned}
& \leqslant C 2^{-k n}\left\|f_{j}\right\|_{L^{q(\cdot)}\left(\mathbb{R}^{n}\right)}\left\{\left|b(x)-b_{B_{j}}\right|^{m}\right. \\
& \cdot\left\|\Omega(x-\cdot) \chi_{j}(\cdot)\right\|_{L^{q^{\prime}(\cdot)}\left(\mathbb{R}^{n}\right)} \\
& \left.+\left\|\Omega(x-\cdot)\left(b_{B_{j}}-b(\cdot)\right)^{m} \chi_{j}(\cdot)\right\|_{L^{q^{\prime}(\cdot)\left(\mathbb{R}^{n}\right)}}\right\} .
\end{aligned}
$$

So we have

$$
\begin{aligned}
& \left|\left[b^{m}, \mu_{\Omega}^{\rho}\right]\left(f_{j}\right)(x)\right| \leqslant C 2^{-k n}\left\|f_{j}\right\|_{L^{q(\cdot)}\left(\mathbb{R}^{n}\right)} \\
& \cdot\left\{\left|b(x)-b_{B_{j}}\right|^{m}\left\|\Omega(x-\cdot) \chi_{j}(\cdot)\right\|_{L^{q^{(} \cdot()\left(\mathbb{R}^{n}\right)}}\right. \\
& \left.+\left\|\Omega(x-\cdot)\left(b_{B_{j}}-b(\cdot)\right)^{m} \chi_{j}(\cdot)\right\|_{L^{q^{(\cdot)}\left(\mathbb{R}^{n}\right)}}\right\} .
\end{aligned}
$$

Noting $s>q^{\prime-}$, we denote $\widetilde{q^{\prime}}(\cdot)>1$ and $1 / q^{\prime}(x)=$ $1 / \widetilde{q^{\prime}}(x)+1 / s$. By Lemmas 8 and 9 we have

$$
\begin{aligned}
& \left\|\Omega(x-\cdot) \chi_{j}(\cdot)\right\|_{L^{q^{\prime}(\cdot)\left(\mathbb{R}^{n}\right)}} \\
& \leqslant\left\|\Omega(x-\cdot) \chi_{j}(\cdot)\right\|_{L^{s}\left(\mathbb{R}^{n}\right)}\left\|\chi_{j}(\cdot)\right\|_{L^{q^{\prime}(\cdot)}\left(\mathbb{R}^{n}\right)} \\
& \leqslant\left\|\Omega(x-\cdot) \chi_{j}(\cdot)\right\|_{L^{s}\left(\mathbb{R}^{n}\right)}\left\|\chi_{B_{j}}\right\|_{L^{q^{\top}(\cdot)}\left(\mathbb{R}^{n}\right)} \\
& \leqslant C 2^{-j v}\left(\int_{A_{j}}|\Omega(x-y)|^{s}|y|^{s \nu} d y\right)^{1 / s}\left\|\chi_{B_{j}}\right\|_{L^{q^{\top}(\cdot)}\left(\mathbb{R}^{n}\right)} \\
& \leqslant C 2^{-j v} 2^{k(\nu+n / s)}\|\Omega\|_{L^{s}\left(S^{n-1}\right)}\left\|\chi_{B_{j}}\right\|_{L^{q^{\top} \cdot(\cdot)}\left(\mathbb{R}^{n}\right)} .
\end{aligned}
$$

When $\left|B_{j}\right| \leqslant 2^{n}$ and $x_{j} \in B_{j}$, by Lemma 10 we have

$$
\left\|\chi_{B_{j}}\right\|_{L^{\widetilde{q}^{\top}(\cdot)\left(\mathbb{R}^{n}\right)}} \approx\left|B_{j}\right|^{1 / \widetilde{q^{\prime}}\left(x_{j}\right)} \approx\left\|\chi_{B_{j}}\right\|_{L^{q^{\prime}(\cdot)\left(\mathbb{R}^{n}\right)}}\left|B_{j}\right|^{-1 / s} .
$$

When $\left|B_{j}\right| \geqslant 1$ we have

$$
\left\|\chi_{B_{j}}\right\|_{L^{q^{\prime}(\cdot)}\left(\mathbb{R}^{n}\right)} \approx\left|B_{j}\right|^{1 / \widetilde{q^{\prime}}(\infty)} \approx\left\|\chi_{B_{j}}\right\|_{L^{q^{\prime}(\cdot)}\left(\mathbb{R}^{n}\right)}\left|B_{j}\right|^{-1 / s} .
$$

So we obtain $\left\|\chi_{B_{j}}\right\|_{L^{q^{q^{\prime}(\cdot)}\left(\mathbb{R}^{n}\right)}} \approx\left\|\chi_{B_{j}}\right\|_{L^{q^{\prime}(\cdot)\left(\mathbb{R}^{n}\right)}}\left|B_{j}\right|^{-1 / s}$.

So we have

$$
\begin{aligned}
& \left\|\Omega(x-\cdot) \chi_{j}(\cdot)\right\|_{L^{q^{\prime}(\cdot)}\left(\mathbb{R}^{n}\right)} \\
& \quad \leqslant C 2^{(k-j)(\nu+n / s)}\|\Omega\|_{L^{s}\left(S^{n-1}\right)}\left\|\chi_{B_{j}}\right\|_{L^{q^{\prime} \cdot(\cdot)\left(\mathbb{R}^{n}\right)}} .
\end{aligned}
$$

Similarly, by Lemma 14 we have

$$
\begin{aligned}
& \left\|\Omega(x-\cdot)\left(b_{B_{j}}-b(\cdot)\right)^{m} \chi_{j}(\cdot)\right\|_{L^{q^{\prime}(\cdot)}\left(\mathbb{R}^{n}\right)} \\
& \leqslant\left\|\Omega(x-\cdot) \chi_{j}(\cdot)\right\|_{L^{s}\left(\mathbb{R}^{n}\right)}\left\|\left(b_{B_{j}}-b(\cdot)\right)^{m} \chi_{j}(\cdot)\right\|_{L^{q^{\prime}(\cdot)}\left(\mathbb{R}^{n}\right)} \\
& \leqslant C\|b\|_{*}^{m}\left\|\chi_{B_{j}}\right\|_{L^{q^{(}(\cdot)}\left(\mathbb{R}^{n}\right)}\left\|\Omega(x-\cdot) \chi_{j}(\cdot)\right\|_{L^{s}\left(\mathbb{R}^{n}\right)} \\
& \leqslant C\|b\|_{*}^{m} 2^{(k-j)(v+n / s)}\|\Omega\|_{L^{s}\left(S^{n-1}\right)}\left\|\chi_{B_{j}}\right\|_{L^{q^{\prime}(\cdot)\left(\mathbb{R}^{n}\right)}} \cdot
\end{aligned}
$$

By (62), (63), Lemmas 3, 4, and 14 we have

$$
\begin{aligned}
& \left\|\left[b^{m}, \mu_{\Omega}^{\rho}\right]\left(f_{j}\right) \chi_{k}\right\|_{L^{q(\cdot)}\left(\mathbb{R}^{n}\right)} \leqslant C 2^{-k n}\left\|f_{j}\right\|_{L^{q(\cdot)}\left(\mathbb{R}^{n}\right)} \\
& \cdot\left(2^{(k-j)(v+n / s)}\|\Omega\|_{L^{s}\left(S^{n-1}\right)}\left\|\chi_{B_{j}}\right\|_{L^{q^{\prime}(\cdot)}\left(\mathbb{R}^{n}\right)}\right. \\
& \times\left\|\left(b(\cdot)-b_{B_{j}}\right)^{m} \chi_{B_{k}}(\cdot)\right\|_{L^{q(\cdot)}\left(\mathbb{R}^{n}\right)}+\|b\|_{*}^{m} \\
& \left.\cdot 2^{(k-j)(\nu+n / s)}\|\Omega\|_{L^{s}\left(S^{n-1}\right)}\left\|\chi_{B_{j}}\right\|_{L^{q^{\prime} \cdot(\cdot)}\left(\mathbb{R}^{n}\right)}\left\|\chi_{k}\right\|_{L^{q(\cdot)}\left(\mathbb{R}^{n}\right)}\right) \\
& \leqslant C 2^{-k n}\left\|f_{j}\right\|_{L^{q(\cdot)}\left(\mathbb{R}^{n}\right)}\left((k-j)^{m}\|b\|_{*}^{m}\right. \\
& \cdot 2^{(k-j)(\nu+n / s)}\|\Omega\|_{L^{s}\left(S^{n-1}\right)} \times\left\|\chi_{B_{j}}\right\|_{L^{q^{\prime} \cdot(\cdot)}\left(\mathbb{R}^{n}\right)}\left\|\chi_{B_{k}}\right\|_{L^{q(\cdot)}\left(\mathbb{R}^{n}\right)} \\
& +\|b\|_{*}^{m} 2^{(k-j)(v+n / s)}\|\Omega\|_{L^{s}\left(S^{n-1}\right)}\left\|\chi_{B_{j}}\right\|_{L^{q^{\prime}(\cdot)\left(\mathbb{R}^{n}\right)}} \\
& \left.\cdot\left\|\chi_{B_{k}}\right\|_{L^{q(\cdot)}\left(\mathbb{R}^{n}\right)}\right) \leqslant C(k-j)^{m}\|b\|_{*}^{m}\left\|f_{j}\right\|_{L^{q \cdot \cdot)}\left(\mathbb{R}^{n}\right)} \\
& \cdot 2^{-k n} 2^{(k-j)(v+n / s)}\|\Omega\|_{L^{s}\left(S^{n-1}\right)} \times\left\|\chi_{B_{j}}\right\|_{L^{q^{\prime}(\cdot)}\left(\mathbb{R}^{n}\right)} \\
& \cdot\left\|\chi_{B_{k}}\right\|_{L^{q(\cdot)}\left(\mathbb{R}^{n}\right)} \leqslant C(k-j)^{m}\|b\|_{*}^{m}\left\|f_{j}\right\|_{L^{q(\cdot)}\left(\mathbb{R}^{n}\right)} \\
& \cdot 2^{(k-j)(\nu+n / s)}\|\Omega\|_{L^{s}\left(S^{n-1}\right)} \frac{\left\|\chi_{B_{j}}\right\|_{L^{q^{\prime}(\cdot)\left(\mathbb{R}^{n}\right)}}}{\left\|\chi_{B_{k}}\right\|_{L^{q^{\prime}(\cdot)\left(\mathbb{R}^{n}\right)}}} \\
& \leqslant C 2^{(j-k)\left(n \delta_{2}-\nu-n / s\right)}(k-j)^{m}\|b\|_{*}^{m}\|\Omega\|_{L^{s}\left(S^{n-1}\right)} \\
& \text { - }\left\|f_{j}\right\|_{L^{q(\cdot)}\left(\mathbb{R}^{n}\right)} \text {. }
\end{aligned}
$$

Thus we obtain

$$
\begin{aligned}
J_{1} \leqslant & C\left\{\sum _ { k = - \infty } ^ { \infty } 2 ^ { k \alpha p } \left(\sum_{j=-\infty}^{k-2} 2^{(j-k)\left(n \delta_{2}-v-n / s\right)}(k-j)^{m}\right.\right. \\
& \left.\left.\times\|b\|_{*}^{m}\|\Omega\|_{L^{s}\left(S^{n-1}\right)}\left\|f_{j}\right\|_{L^{q(\cdot)}\left(\mathbb{R}^{n}\right)}\right)^{p}\right\}^{1 / p}=C\|b\|_{*}^{m} \\
& \cdot\|\Omega\|_{L^{s}\left(S^{n-1}\right)} \\
& \cdot\left\{\sum _ { k = - \infty } ^ { \infty } \left(\sum_{j=-\infty}^{k-2} 2^{j \alpha} 2^{(j-k)\left(n \delta_{2}-\gamma-n / s-\alpha\right)}(k-j)^{m}\right.\right. \\
& \left.\left.\times\left\|f_{j}\right\|_{L^{q(\cdot)}\left(\mathbb{R}^{n}\right)}\right)^{p}\right\}^{1 / p} .
\end{aligned}
$$

If $1<p<\infty$, take $1 / p+1 / p^{\prime}=1$. Since $n \delta_{2}-\nu-n / s-\alpha>0$, by the Hölder inequality we have

$$
\begin{aligned}
J_{1} \leqslant & C\|b\|_{*}^{m}\|\Omega\|_{L^{s}\left(S^{n-1}\right)} \\
& \times\left\{\sum_{k=-\infty}^{\infty}\left(\sum_{j=-\infty}^{k-2} 2^{j \alpha p}\left\|f_{j}\right\|_{L^{q \cdot \cdot}\left(\mathbb{R}^{n}\right)}^{p} 2^{(j-k)\left(n \delta_{2}-\gamma-n / s-\alpha\right) p / 2}\right)\right.
\end{aligned}
$$


Journal of Function Spaces

9

$$
\begin{aligned}
& \left.\times\left(\sum_{j=-\infty}^{k-2} 2^{(j-k)\left(n \delta_{2}-\gamma-n / s-\alpha\right) p^{\prime} / 2}(k-j)^{m p^{\prime}}\right)^{p / p^{\prime}}\right\}^{1 / p} \\
& \leqslant C\|b\|_{*}^{m}\|\Omega\|_{L^{s}\left(S^{n-1}\right)} \\
& \times\left\{\sum_{k=-\infty}^{\infty}\left(\sum_{j=-\infty}^{k-2} 2^{j \alpha p}\left\|f_{j}\right\|_{L^{q(\cdot)}\left(\mathbb{R}^{n}\right)}^{p} 2^{(j-k)\left(n \delta_{2}-\gamma-n / s-\alpha\right) p / 2}\right)\right\}^{1 / p} \\
& =C\|b\|_{*}^{m}\|\Omega\|_{L^{s}\left(S^{n-1}\right)} \times\left\{\sum_{j=-\infty}^{\infty} 2^{j \alpha p}\left\|f_{j}\right\|_{L^{q(\cdot)}\left(\mathbb{R}^{n}\right)}^{p}\right. \\
& \left.\cdot\left(\sum_{k=j+2}^{\infty} 2^{(j-k)\left(n \delta_{2}-\gamma-n / s-\alpha\right) p / 2}\right)\right\}^{1 / p} \leqslant C\|b\|_{*}^{m} \\
& \cdot\left\{\sum_{j=-\infty}^{\infty} 2^{j \alpha p}\left\|f_{j}\right\|_{L^{q(\cdot)}}^{p}\right\}^{1 / p}=C\|b\|_{*}^{m}\|f\|_{\dot{K}_{q(\cdot)}^{\alpha, p}\left(\mathbb{R}^{n}\right)} \cdot
\end{aligned}
$$

If $0<p \leqslant 1$, then we have

$$
\begin{aligned}
J_{1} \leqslant & C\|b\|_{*}^{m}\|\Omega\|_{L^{s}\left(\mathrm{~S}^{n-1}\right)} \\
& \times\left\{\sum_{k=-\infty}^{\infty} \sum_{j=-\infty}^{k-2} 2^{j \alpha p} 2^{(j-k)\left(n \delta_{2}-v-n / s-\alpha\right) p}(k-j)^{m p}\right. \\
& \left.\cdot\left\|f_{j}\right\|_{L^{q(\cdot)}\left(\mathbb{R}^{n}\right)}^{p}\right\}^{1 / p}=C\|b\|_{*}^{m}\|\Omega\|_{L^{s}\left(S^{n-1}\right)} \\
& \times\left\{\sum_{j=-\infty}^{\infty} 2^{j \alpha p}\left\|f_{j}\right\|_{L^{q(\cdot)}\left(\mathbb{R}^{n}\right)}^{p}\right. \\
& \left.\cdot\left(\sum_{k=j+2}^{\infty} 2^{(j-k)\left(n \delta_{2}-v-n / s-\alpha\right) p}(k-j)^{m p}\right)\right\}^{1 / p} \\
& \leqslant C\|b\|_{*}^{m}\|f\|_{\dot{K}_{q(\cdot)}^{\alpha, p}\left(\mathbb{R}^{n}\right)} \cdot
\end{aligned}
$$

Let us now estimate $J_{3}$. Note that $x \in A_{k}, y \in A_{j}$, and $j \geqslant k+2$, so we have $|x-y| \sim|y|$. We consider

$$
\begin{aligned}
\left|\left[b^{m}, \mu_{\Omega}^{\rho}\right]\left(f_{j}\right)(x)\right| \leqslant & \left(\int_{0}^{|y|}\left|\int_{|x-y| \leqslant t} \frac{\Omega(x-y)}{|x-y|^{n-\rho}}[b(x)-b(y)]^{m} f_{j}(y) d y\right|^{2} \frac{d t}{t^{2 \rho+1}}\right)^{1 / 2} \\
& +\left(\int_{|y|}^{\infty}\left|\int_{|x-y| \leqslant t} \frac{\Omega(x-y)}{|x-y|^{n-\rho}}[b(x)-b(y)]^{m} f_{j}(y) d y\right|^{2} \frac{d t}{t^{2 \rho+1}}\right)^{1 / 2}=: J_{31}+J_{32} .
\end{aligned}
$$

Similar to the estimate for $J_{11}$, we get

$$
\begin{aligned}
J_{31} & \leqslant C 2^{(k-j) / 2} 2^{-j n}\left\|f_{j}\right\|_{L^{q(\cdot)}\left(\mathbb{R}^{n}\right)} \\
& \cdot\left\{\left|b(x)-b_{B_{k}}\right|^{m}\left\|\Omega(x-\cdot) \chi_{j}(\cdot)\right\|_{L^{q^{\prime}(\cdot)\left(\mathbb{R}^{n}\right)}}\right. \\
& \left.+\left\|\Omega(x-\cdot)\left(b_{B_{k}}-b(\cdot)\right)^{m} \chi_{j}(\cdot)\right\|_{L^{q^{(\cdot)}\left(\mathbb{R}^{n}\right)}}\right\} .
\end{aligned}
$$

Similar to the estimate for $J_{12}$, we get

$$
\begin{aligned}
J_{32} & \leqslant C 2^{-j n}\left\|f_{j}\right\|_{L^{q(\cdot)}\left(\mathbb{R}^{n}\right)} \\
& \cdot\left\{\left|b(x)-b_{B_{k}}\right|^{m}\left\|\Omega(x-\cdot) \chi_{j}(\cdot)\right\|_{L^{q^{\prime}(\cdot)}\left(\mathbb{R}^{n}\right)}\right. \\
& \left.+\left\|\Omega(x-\cdot)\left(b_{B_{k}}-b(\cdot)\right)^{m} \chi_{j}(\cdot)\right\|_{L^{q^{\prime}(\cdot)\left(\mathbb{R}^{n}\right)}}\right\} .
\end{aligned}
$$

So we have

$$
\begin{aligned}
& \left|\left[b^{m}, \mu_{\Omega}^{\rho}\right]\left(f_{j}\right)(x)\right| \leqslant C 2^{-j n}\left\|f_{j}\right\|_{L^{q(\cdot)}\left(\mathbb{R}^{n}\right)} \\
& \cdot\left\{\left|b(x)-b_{B_{k}}\right|^{m}\left\|\Omega(x-\cdot) \chi_{j}(\cdot)\right\|_{L^{q^{\prime}(\cdot)\left(\mathbb{R}^{n}\right)}}\right. \\
& \left.+\left\|\Omega(x-\cdot)\left(b_{B_{k}}-b(\cdot)\right)^{m} \chi_{j}(\cdot)\right\|_{L^{q^{\prime}(\cdot)}\left(\mathbb{R}^{n}\right)}\right\} .
\end{aligned}
$$

By (62), (63), Lemmas 3, 4, and 14 we have

$$
\begin{aligned}
& \left\|\left[b^{m}, \mu_{\Omega}^{\rho}\right]\left(f_{j}\right) \chi_{k}\right\|_{L^{q(\cdot)}\left(\mathbb{R}^{n}\right)} \leqslant C 2^{-j n}\left\|f_{j}\right\|_{L^{q(\cdot)}\left(\mathbb{R}^{n}\right)}\left(\|b\|_{*}^{m}\right. \\
& \cdot 2^{(k-j)(\nu+n / s)} \times\|\Omega\|_{L^{s}\left(S^{n-1}\right)}\left\|\chi_{j}\right\|_{L^{q^{\prime}(\cdot)\left(\mathbb{R}^{n}\right)}}\left\|\chi_{B_{k}}\right\|_{L^{q(\cdot)}\left(\mathbb{R}^{n}\right)} \\
& +2^{(k-j)(\nu+n / s)}\|\Omega\|_{L^{s}\left(S^{n-1}\right)} \\
& \left.\cdot\left\|\left(b_{B_{k}}-b(\cdot)\right)^{m} \chi_{B_{j}}(\cdot)\right\|_{L^{q^{\prime}(\cdot)}\left(\mathbb{R}^{n}\right)}\left\|\chi_{k}\right\|_{L^{q(\cdot)}\left(\mathbb{R}^{n}\right)}\right) \\
& \leqslant C 2^{-j n}\left\|f_{j}\right\|_{L^{q \cdot \cdot(}\left(\mathbb{R}^{n}\right)}\left(\|b\|_{*}^{m} 2^{(k-j)(v+n / s)} \times\|\Omega\|_{L^{s}\left(S^{n-1}\right)}\right. \\
& \cdot\left\|\chi_{B_{j}}\right\|_{L^{q^{(\cdot)}\left(\mathbb{R}^{n}\right)}}\left\|\chi_{B_{k}}\right\|_{L^{q(\cdot)}\left(\mathbb{R}^{n}\right)}+(j-k)^{m}\|b\|_{*}^{m} \\
& \left.\cdot 2^{(k-j)(v+n / s)}\|\Omega\|_{L^{s}\left(S^{n-1}\right)}\left\|\chi_{B_{j}}\right\|_{L^{q^{\prime}(\cdot)}\left(\mathbb{R}^{n}\right)}\left\|\chi_{B_{k}}\right\|_{L^{q(\cdot)}\left(\mathbb{R}^{n}\right)}\right) \\
& \leqslant C(j-k)^{m}\|b\|_{*}^{m}\left\|f_{j}\right\|_{L^{q(\cdot)}\left(\mathbb{R}^{n}\right)} 2^{(k-j)(v+n / s)}\|\Omega\|_{L^{s}\left(S^{n-1}\right)} \\
& \cdot \frac{\left\|\chi_{B_{k}}\right\|_{L^{q(\cdot)}\left(\mathbb{R}^{n}\right)}}{\left\|\chi_{B_{j}}\right\|_{L^{q \cdot(\cdot)}\left(\mathbb{R}^{n}\right)}} \leqslant C 2^{(k-j)\left(n \delta_{1}+\nu+n / s\right)}(j-k)^{m}\|b\|_{*}^{m} \\
& \cdot\|\Omega\|_{L^{s}\left(S^{n-1}\right)}\left\|f_{j}\right\|_{L^{q(\cdot)}\left(\mathbb{R}^{n}\right)} \cdot
\end{aligned}
$$


Thus we obtain

$$
\begin{aligned}
J_{3} & \leqslant C\|b\|_{*}^{m}\|\Omega\|_{L^{s}\left(S^{n-1}\right)} \times\left\{\sum_{k=-\infty}^{\infty} 2^{k \alpha p}\right. \\
& \left.\cdot\left(\sum_{j=k+2}^{\infty} 2^{(k-j)\left(n \delta_{1}+\nu+n / s\right)}(j-k)^{m}\left\|f_{j}\right\|_{L^{q(\cdot)}\left(\mathbb{R}^{n}\right)}\right)^{p}\right\}^{1 / p} \\
& =C\|b\|_{*}^{m}\|\Omega\|_{L^{s}\left(S^{n-1}\right)} \times\left\{\sum _ { k = - \infty } ^ { \infty } \left(\sum_{j=k+2}^{\infty} 2^{j \alpha}\right.\right. \\
& \left.\left.\cdot 2^{(k-j)\left(n \delta_{1}+\nu+n / s+\alpha\right)}(j-k)^{m}\left\|f_{j}\right\|_{L^{q(\cdot)}\left(\mathbb{R}^{n}\right)}\right)^{p}\right\}^{1 / p} .
\end{aligned}
$$

If $1<p<\infty$, take $1 / p+1 / p^{\prime}=1$. Since $n \delta_{1}+v+n / s+\alpha>0$, by the Hölder inequality we have

$$
\begin{aligned}
J_{3} & \leqslant C\|b\|_{*}^{m}\|\Omega\|_{L^{s}\left(S^{n-1}\right)} \times\left\{\sum _ { k = - \infty } ^ { \infty } \left(\sum_{j=k+2}^{\infty} 2^{j \alpha p}\right.\right. \\
& \left.\cdot\left\|f_{j}\right\|_{L^{q(\cdot)}\left(\mathbb{R}^{n}\right)}^{p} 2^{(k-j)\left(n \delta_{1}+\nu+n / s+\alpha\right) p / 2}\right) \\
& \left.\times\left(\sum_{j=k+2}^{\infty} 2^{(k-j)\left(n \delta_{1}+\nu+n / s+\alpha\right) p^{\prime} / 2}(j-k)^{m p^{\prime}}\right)^{p / p^{\prime}}\right\}^{1 / p} \\
& \leqslant C\|b\|_{*}^{m}\|\Omega\|_{L^{s}\left(S^{n-1}\right)} \times\left\{\sum_{j=-\infty}^{\infty} 2^{j \alpha p}\left\|f_{j}\right\|_{L^{q(\cdot)}\left(\mathbb{R}^{n}\right)}^{p}\right. \\
& \cdot\left(\sum_{k=-\infty}^{j-2} 2^{(k-j)\left(n \delta_{1}+v+n / s+\alpha\right) p / 2}\right\}^{1 / p} \leqslant C\|b\|_{*}^{m} \\
& \cdot\left\{\sum_{j=-\infty}^{\infty} 2^{j \alpha p}\left\|f_{j}\right\|_{L^{q(\cdot)}\left(\mathbb{R}^{n}\right)}^{p}\right\}^{1 / p}=C\|b\|_{*}^{m}\|f\|_{\dot{K}_{q(\cdot)}^{\alpha, p}\left(\mathbb{R}^{n}\right)} .
\end{aligned}
$$

If $0<p \leqslant 1$, then we have

$$
\begin{aligned}
J_{3} & \leqslant C\|b\|_{*}^{m}\|\Omega\|_{L^{s}\left(S^{n-1}\right)} \times\left\{\sum_{j=-\infty}^{\infty} 2^{j \alpha p}\left\|f_{j}\right\|_{L^{q(\cdot)}\left(\mathbb{R}^{n}\right)}^{p}\right. \\
& \left.\cdot\left(\sum_{k=-\infty}^{j-2} 2^{(k-j)\left(n \delta_{1}+v+n / s+\alpha\right) p}(j-k)^{m p}\right)\right\}^{1 / p} \\
& \leqslant C\|b\|_{*}^{m}\|f\|_{\dot{K}_{q(\cdot)}^{\alpha, p}\left(\mathbb{R}^{n}\right)} .
\end{aligned}
$$

Thus by (53), (54) and (66), (67), (74), and (75) we complete the proof of Theorem 13.

\section{Data Availability}

No data were used to support this study.

\section{Conflicts of Interest}

The authors declare that there are no conflicts of interest regarding the publication of this paper.

\section{Acknowledgments}

This work is supported by China Postdoctoral Science Foundation Funded Project (Grant no. 2016M601105), Shandong Provincial Natural Science Foundation, China (Grant no. ZR2017MA041), a Project of Shandong Province Higher Educational Science and Technology Program (Grant no. J18KA225), and National Natural Science Foundation of China (Grant no. 11761026).

\section{References}

[1] O. Kováčik and J. Rákosník, "On spaces $L^{p(x)}$ and $W^{k, p(x), "}$ Czechoslovak Mathematical Journal, vol. 41, pp. 592-618, 1991.

[2] C. Capone, D. Cruz-Uribe, and A. Fiorenza, "The fractional maximal operator and fractional integrals on variable $L^{p}$ spaces," Revista Matemática Iberoamericana, vol. 23, no. 3, pp. 743-770, 2007.

[3] D. V. Cruz-Uribe and A. Fiorenza, Variable Lebesgue Spaces: Foundations and Harmonic Analysis, Applied and Numerical Harmonic Analysis, Birkhäuser, Basel, Switzerland, 2013.

[4] D. Cruz-Uribe, A. Fiorenza, J. M. Martell, and C. Pérez, "The boundedness of classical operators on variable $L^{p}$ spaces," Annales Academioe Scientiarium Fennice Mathematica, vol. 31, pp. 239-264, 2006.

[5] L. Diening, P. Harjulehto, P. Hästö, and M. Růžička, "Lebesgue and Sobolev spaces with variable exponents," Lecture Notes in Mathematics, vol. 2017, Springer, Heidelberg, 2011.

[6] H. Wang, Z. Fu, and Z. Liu, "Higher-order commutators of Marcinkiewicz integrals on variable Lebesgue spaces," Acta Mathematica Scientia, Series A, vol. 32, pp. 1092-1101, 2012.

[7] M. Izuki, "Boundedness of sublinear operators on Herz spaces with variable exponent and application to wavelet characterization," Analysis Mathematica, vol. 36, no. 1, pp. 33-50, 2010.

[8] H. Wang and Z. Liu, "The Herz-type Hardy spaces with variable exponent and their applications," Taiwanese Journal of Mathematics, vol. 16, no. 4, pp. 1363-1389, 2012.

[9] E. M. Stein, "On the functions of Littlewood-Paley, Lusin, and Marcinkiewicz," Transactions of the American Mathematical Society, vol. 88, pp. 430-466, 1958.

[10] Z. Liu and H. Wang, "Boundedness of Marcinkiewicz integrals on Herz spaces with variable exponent," Jordan Journal of Mathematics and Statistics, vol. 5, no. 4, pp. 223-239, 2012.

[11] J. Tan and Z. Liu, "Some boundedness of homogeneous fractional integrals on variable exponent function spaces," Acta Mathematica Sinica, Chinese Series, vol. 58, pp. 309-320, 2015.

[12] X. Shi and Y. Jiang, "Weighted boundedness of parametric Marcinkiewicz integral and higher order commutator," Analysis in Theory and Applications, vol. 25, no. 1, pp. 25-39, 2009.

[13] B. Muckenhoupt and R. L. Wheeden, "Weighted norm inequalities for singular and fractional integrals," Transactions of the American Mathematical Society, vol. 161, pp. 249-258, 1971. 
[14] E. Nakai and Y. Sawano, "Hardy spaces with variable exponents and generalized Campanato spaces," Journal of Functional Analysis, vol. 262, no. 9, pp. 3665-3748, 2012.

[15] M. Izuki, "Boundedness of commutators on Herz spaces with variable exponent," Rendiconti del Circolo Matematico di Palermo, vol. 59, no. 2, pp. 199-213, 2010. 


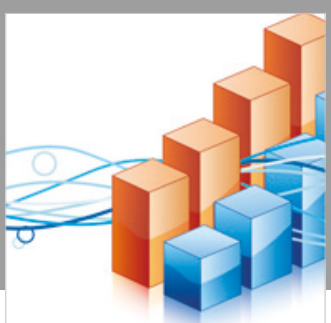

Advances in

Operations Research

\section{-n-m}
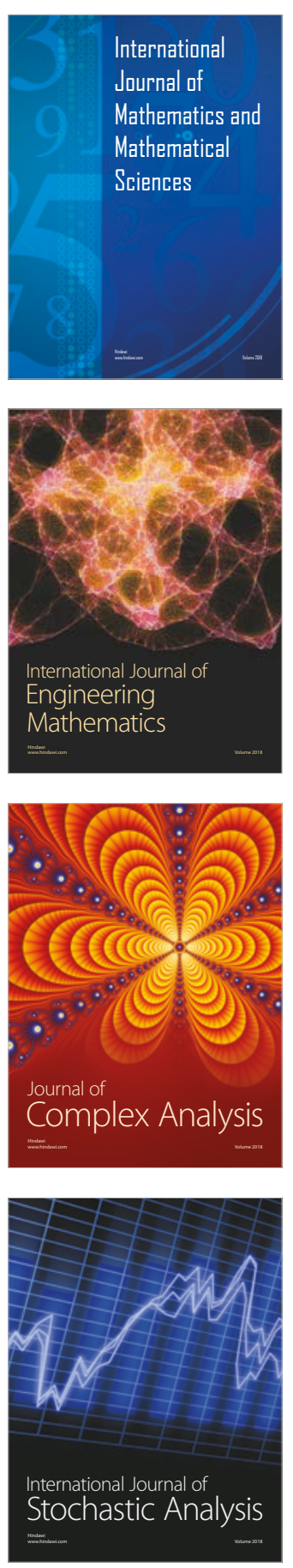
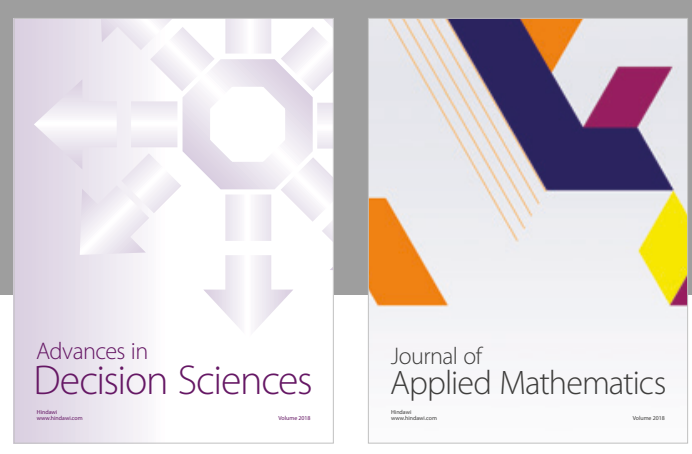

Journal of

Applied Mathematics
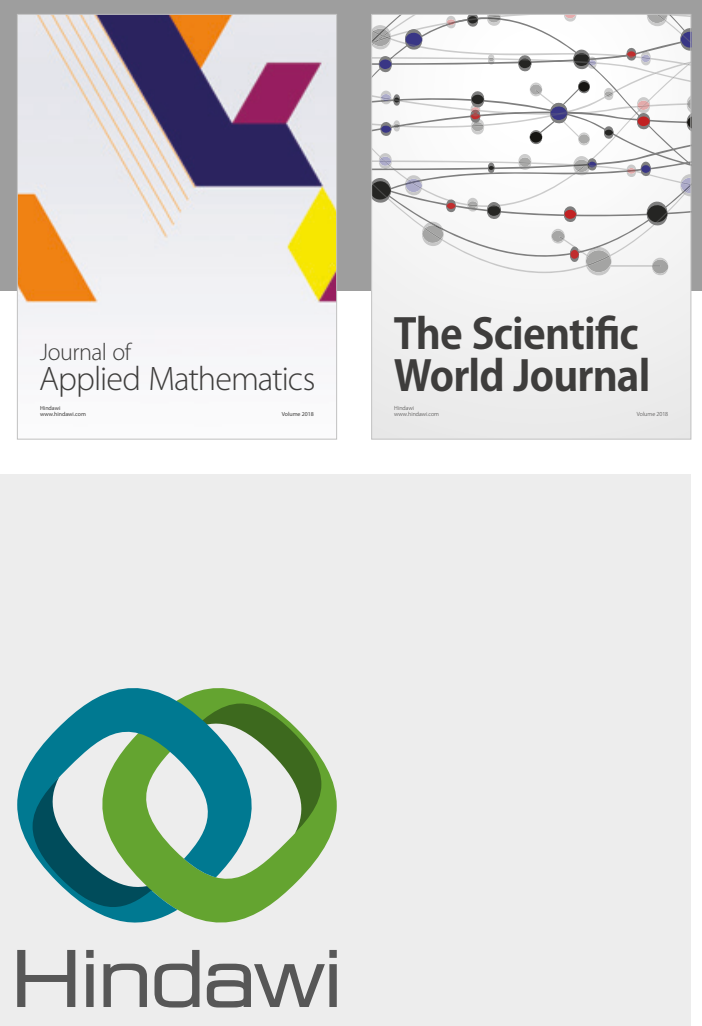

Submit your manuscripts at

www.hindawi.com

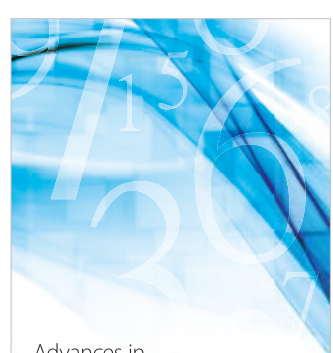

Advances in
Numerical Analysis
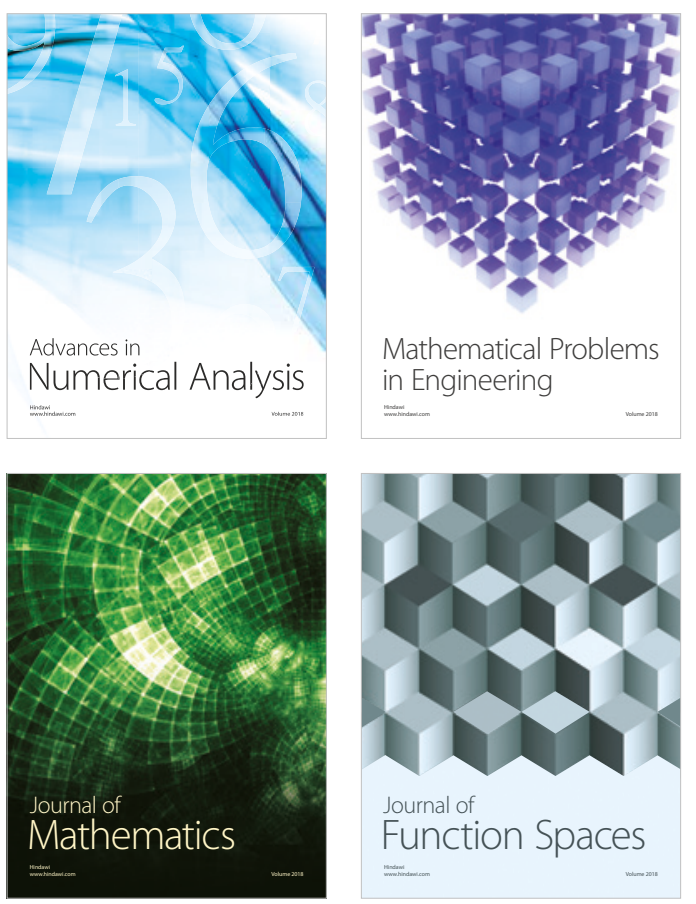

Mathematical Problems in Engineering

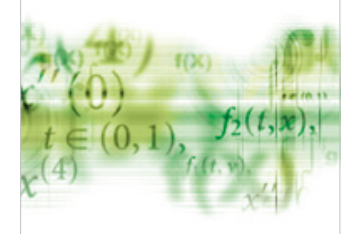

International Journal of

Differential Equations

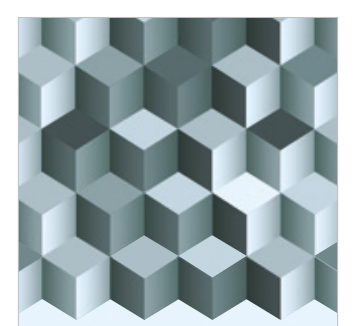

Journal of

Function Spaces

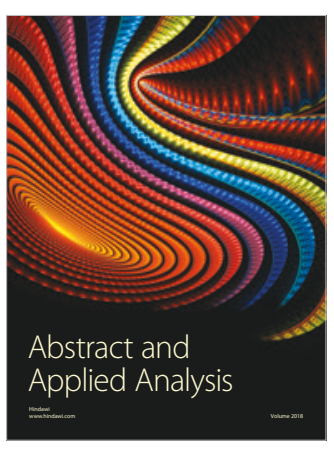

The Scientific

World Journal

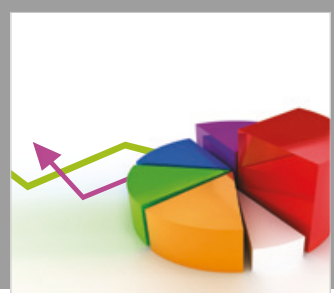

Journal of

Probability and Statistics
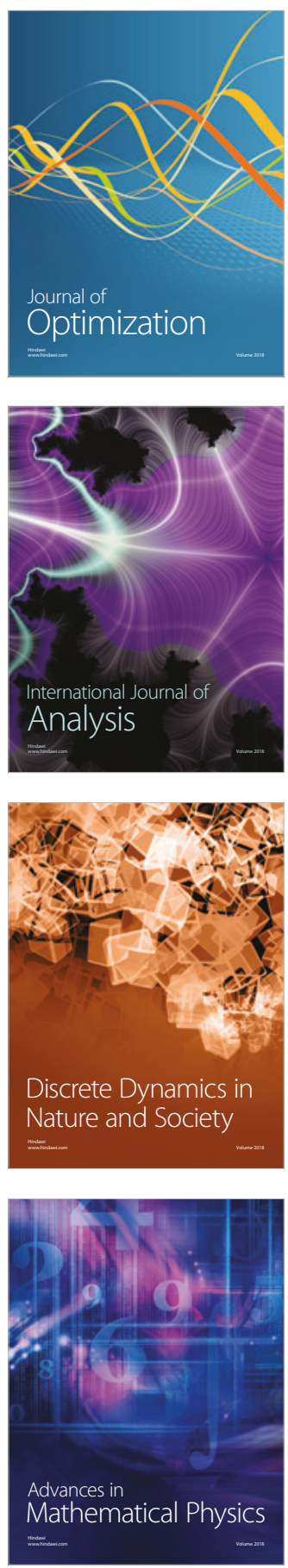